\title{
A Mulli-Modal Approach to Closing Exploratory Laparotomies Including High-Risk Wounds
}

\author{
Erin G. Andrade ${ }^{1}$, Jarot J. Guerra ${ }^{2}$, Laurie Punch ${ }^{3}$ \\ 1. Surgery, Washington University, St. Louis, USA 2. Surgery, Rutgers New Jersey Medical School, Newark, USA 3. \\ Surgery, Barnes-Jewish Hospital, Washington University, St. Louis, USA
}

Corresponding author: Erin G. Andrade, andradee@wustl.edu

\section{Abstract \\ Background}

Laparotomy incisions with contamination have a high incidence of surgical site infection (SSI). One strategy to reduce SSI has been to allow these wounds to heal by secondary intention; however, this results in an ongoing need for wound care after discharge.

\section{Methods}

A prospectively maintained Acute and Critical Care Surgery database was queried for patients who underwent exploratory laparotomy during 2008-2018. Patients were stratified into two groups: 2008-2015 (no protocol [NP]) and 2016-2018 (closure protocol [CP]). CP patients were operated on by a single surgeon utilizing a multi-modal high-risk incisional closure protocol, which included dilute chlorhexidine lavage, closed suction drains for incisions deeper than 3 centimeters, and incisional negative-pressure wound therapy (iNPWT). The CDC (Centers for Disease Control and Prevention) guidelines were used to determine wound classification and SSI based on chart review. Groups were compared using univariate and multivariate analysis.

\section{Results}

A total of 139 patients met the study criteria. The overall SSI rate, including superficial and deep space infections, was no different in NP versus CP (21.6 vs. $24.1 \%$; $\mathrm{p}=0.74)$. The rate of superficial SSI was similar between NP and CP (11.8 vs. 8.4\%; $\mathrm{p}=0.53$ ). Rates of wound closure at discharge were higher in the CP group than the NP group across wound classes, with the greatest difference among dirty wounds (50.0\% NP vs. 94.9\% CP; $\mathrm{p}<0.01)$. CP significantly increased the likelihood of wound closure ( $\mathrm{OR}=179.2 ; \mathrm{p}<0.001)$ even after controlling for body mass index, wound classification, ASA (American Society of Anesthesiologists) status, and initially open abdomen.

\section{Conclusions}

Received 06/09/2020 Review began 06/14/2020 Review ended 07/03/2020 Published 07/09/2020

\section{() Copyright 2020}

Andrade et al. This is an open access article distributed under the terms of the Creative Commons Attribution License CC-BY 4.0., which permits unrestricted use, distribution, and reproduction in any medium, provided the original author and source are credited.
By addressing both tissue factors and bacterial burden through the use of a multi-modal high-risk incisional closure protocol involving iNPWT, all wounds can be considered for closure without increasing the risk of SSI.

Categories: General Surgery, Healthcare Technology

Keywords: surgical site infection, laparotomy, closed incision negative pressure wound therapy, emergent general surgery

\section{Introduction}

Surgical site infections (SSIs) represent both the most prevalent and most costly form of nosocomial infection in the United States, occurring after 2-5\% of all operations and more than $16 \%$ of abdominal operations annually $[1,2]$. Public reporting of SSI incidence rates is a required quality indicator used by the Centers for Medicare and Medicaid Services [3]. It has been estimated that $55 \%$ of SSI are preventable through the use of best practices [4]. Efforts to reduce SSI are common in the surgical literature but vary based on wound classification.

Healing by secondary intention is often used in high-risk wounds to decrease rates of SSI [5]. Wounds may be high-risk for infection secondary to patient factors (age, obesity, smoking, malnutrition, diabetes mellitus), tissue characteristics (ischemia, dead space), and level of bacterial contamination. Although healing by secondary intention reduces SSI, it increases healthcare costs [6,7]. Historically, delayed primary closure has been used to mitigate the risk of SSI in contaminated or dirty wounds by avoiding closure at the initial operation when the bacterial burden is higher, while still eventually achieving a primarily closed wound $[8,9]$. However, not all studies show a decrease in SSI with delayed primary closure $[10,11]$. 
While many have addressed patient factors pre-operatively to reduce SSI rates, this is rarely possible in emergent general surgery cases [12,13]. However, tissue characteristics and level of contamination can be addressed through a multi-modal approach. Prior studies have shown that subcutaneous wound depth and obesity are risk factors for SSI [14-16]. Obesity increases the risk of SSI even in clean and clean-contaminated cases, where SSIs are generally rare [16]. Because patient risk factors, such as wound depth and obesity, may make a wound high-risk for complications beyond the risk typically associated with a given wound classification, we developed a multi-modal protocol that identifies wounds at high-risk for wound complications including SSI and dehiscence across all wound classifications based on the following high-risk criteria: body mass index (BMI) greater than 30, delayed primary closure, creation or revision of an ostomy, or the presence of an enterocutaneous fistula. Our closure protocol seeks to counteract high-risk tissue characteristics through incisional negative-pressure wound therapy (iNPWT), selective use of closed suction drains in deep wounds, and reduction of contamination level through dilute chlorhexidine lavage. The use of iNPWT increases oxygenation through angiogenesis, reduces tension on wound edges, and lowers the risk of seroma formation [17]. In deep wounds, in addition to iNPWT, closed suction drains were added to further decrease seroma risk. Finally, chlorhexidine gluconate, both an effective biocide and a surfactant, was lavaged to decrease the wound's bioburden [18,19].

We hypothesized that the use of the above multi-modal high-risk incisional closure protocol could increase rates of primary closure without increasing rates of SSI.

\section{Materials And Methods}

A prospectively maintained acute care surgery database spanning 2008-2018 was queried for patients who underwent exploratory laparotomy using ICD-9 (International Classification of Diseases, Ninth Revision) codes. Trauma patients and in-hospital deaths were excluded. Patients were stratified into two time periods: 2008-2015 (no protocol [NP]) and 2016-2018 (closure protocol [CP]). In the NP group, all exploratory laparotomies performed by surgeons in the acute care division were included. Patients were closed according to usual practice with staples or subcuticular absorbable suture according to surgeon preference.

Contaminated wounds were lavaged with saline prior to closure. Within the NP group, no iNPWT was used. In the CP period, patients were operated on by a single surgeon, who was piloting the use of a multi-modal incisional closure protocol. Within the CP group, patients were deemed high-risk if they met any of the following criteria: BMI greater than 30, delayed primary closure, creation or revision of an ostomy, or the presence of an enterocutaneous fistula. After fascial closure, high-risk patients underwent dilute chlorhexidine lavage of the soft tissue, placement of subcutaneous closed suction drains for incisions deeper than 3 centimeters, and iNPWT (Figure 1). Incisional NPWT was created using PolyMem WIC Silver as a base over the closed incision, with black foam placed on top.

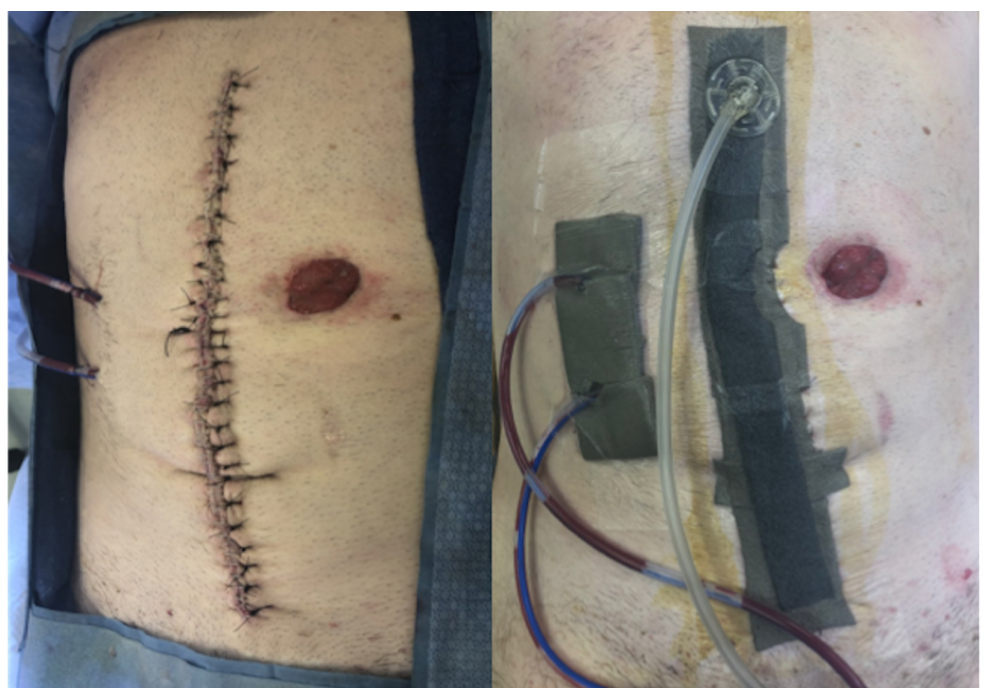

FIGURE 1: Incisional Negative-Pressure Wound Therapy with PolyMem Wic Silver®

Study variables including patient demographics, medical co-morbidities, hospital length of stay, readmissions, wound class, and the presence of SSI were manually extracted from the electronic medical record. The wound classification was determined by review of the operative report and application of the Centers for Disease Control and Prevention (CDC) definition [20]. SSIs were defined as a wound infection occurring within 30 days of surgery and subdivided into superficial incisional, deep incisional, and organ space infections based on the CDC guidelines; for open wounds, superficial SSIs were defined as wounds having erythema or purulence that were determined to require antibiotics by the treating physician [20]. 


\section{Cureus}

Complications were defined as any SSI, return to emergency room, re-admission, or wound dehiscence within 30 days.

Statistical analysis was performed using Stata 14.3 (StataCorp LLC, College Station, TX, USA). Categorical variables were analyzed using Pearson's chi-square test to determine statistical significance. Student's t-test was used to determine statistical significance for continuous variables, which are reported as mean and standard deviation. Multivariate logistic regression models were used to assess the impact of CP on skin closure considering known risk factors for SSI as possible confounders. A step-wise approach was used with any variable, with $\mathrm{p}<0.20$ remaining in the model. $\mathrm{P}$-values less than 0.05 were considered significant.

\section{Results}

During the study period, 170 patients underwent exploratory laparotomy for a non-traumatic indication. After 31 patients were excluded for in-hospital mortality, 52 patients remained in the NP group and 87 in the $\mathrm{CP}$ group. Demographics between the two groups were similar (Table 1). The CP group contained higher risk patients both in terms of higher mean BMI (26.6 vs. 30.6; $\mathrm{p}=0.01$ ) and higher proportion of patients with the American Society of Anesthesiologists (ASA) class III or higher (Table 1). Additionally, the CP group contained a greater proportion of dirty cases compared with the NP group ( $44.8 \%$ vs. $26.9 \%$; $=0.002$; Table 2). Within the NP group, $42.0 \%$ met at least one of the criteria for high-risk closure according to the high-risk incisional closure protocol compared with $67.8 \%$ if the CP group $(\mathrm{p}<0.01)$. Compliance with the high-risk protocol among the CP patients who met high-risk criteria was 84.8\% (50/59); an additional 13 patients who did not meet high-risk criteria also underwent high-risk protocol.

\begin{tabular}{|c|c|c|c|}
\hline Variable & No Protocol, $n=52$ & Closure Protocol, n = 87 & p-Value \\
\hline Age, years (SD) & 57.7 (15.4) & $58.1(16.4)$ & 0.89 \\
\hline Male (n) & $44.2 \%(23)$ & $44.8 \%(39)$ & 0.95 \\
\hline Smoker (n) & $26.5 \%(13)$ & $22.6 \%(19)$ & 0.61 \\
\hline Diabetes mellitus (n) & $17.3 \%(9)$ & $28.7 \%(25)$ & 0.13 \\
\hline Steroid use (n) & $10.2 \%(5)$ & $13.8 \%(12)$ & 0.54 \\
\hline Body mass index (SD) & $26.6(7.1)$ & $30.6(10.0)$ & 0.01 \\
\hline ASA class III or greater (n) & $66.0 \%(33)$ & $85.1 \%(74)$ & 0.01 \\
\hline
\end{tabular}

\section{TABLE 1: Demographic Characteristics and Co-morbidities}

SD, standard deviation; ASA, American Society of Anesthesiologists

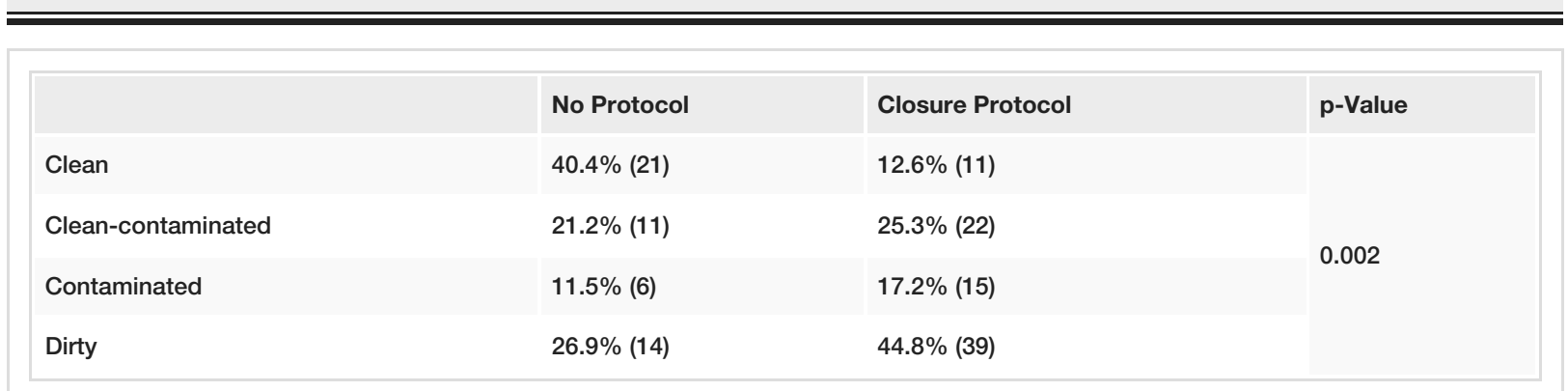

TABLE 2: Case Volume by Wound Classification

Both groups had similar proportions of patients with an open abdomen after their initial operation $(25.0 \%$ NP vs. $27.6 \% \mathrm{CP} ; \mathrm{p}=0.74$; Table 3). Reasons for not closing the fascia at the time of initial operation included hemodynamic instability, marginally ischemic bowel requiring a second look operation, and bowel edema preventing closure. In the NP group, $13.5 \%$ of patients underwent delayed primary closure versus $26.4 \%$ in the $\mathrm{CP}$ group ( $\mathrm{p}=0.07)$. At the time of final operation, $97.7 \%$ in the $\mathrm{CP}$ group had their skin closed compared with $75.0 \%$ in the NP group ( $\mathrm{p}<0.01$; Figure 2). Despite having higher risk patients in the CP group, the overall SSI rate was not significantly different in NP versus CP (21.6\% vs. $24.1 \%$; $\mathrm{p}=0.74)$. This was also true for superficial SSI (11.8\% NP vs. 8.4\% CP; $\mathrm{p}=0.53)$. Thirty-day complication rates were not significantly different between the groups ( $46.2 \% \mathrm{NP}$ vs. $44.1 \% \mathrm{CP} ; \mathrm{p}=0.81$ ). While the NP had a higher rate of emergency 


\section{Cureus}

room visits within 30 days than the CP group (28.9\% vs. $14.9 \%$; $\mathrm{p}=0.05$ ), both groups had relatively high readmission rates, with $28.9 \%$ in the NP group and $21.8 \%$ in the CP group getting readmitted within 30 days $(\mathrm{p}=0.47)$. Stratified by wound class, the CP group had higher rates of wound closure in every wound class (Figure 1). The greatest difference in closure rates was seen in dirty cases. Despite closing high-risk wounds, the CP group had similar rates of superficial skin dehiscence to the NP group (7.6\% vs. 5.1\%; $\mathrm{p}=0.62$ ).

\begin{tabular}{|c|c|c|c|}
\hline Variable & No Protocol & Closure Protocol & p-value \\
\hline Initially open (n) & $25.0 \%(13)$ & $27.6 \%(24)$ & 0.74 \\
\hline Length of stay, days (SD) & $17.4(29.5)$ & $20.9(16.6)$ & 0.38 \\
\hline Any SSI (n) & $21.6 \%(11)$ & $24.1 \%(20)$ & 0.74 \\
\hline Superficial SSI (n) & $11.8 \%(6)$ & $8.4 \%(7)$ & 0.53 \\
\hline Skin closed (n) & $75.0 \%(39)$ & $97.7 \%(85)$ & $<0.01$ \\
\hline 30-day return ER (n) & $28.9 \%(15)$ & $14.9 \%(13)$ & 0.05 \\
\hline 30 day readmission $(\mathrm{n})$ & $28.9 \%(15)$ & $21.8 \%(19)$ & 0.35 \\
\hline 30-day complications (n) & $46.2 \%(24)$ & $44.1 \%(37)$ & 0.81 \\
\hline
\end{tabular}

\section{TABLE 3: Clinical Management and Outcomes}

$\mathrm{SD}$, standard deviation; SSI, surgical site infection; ER, emergency room

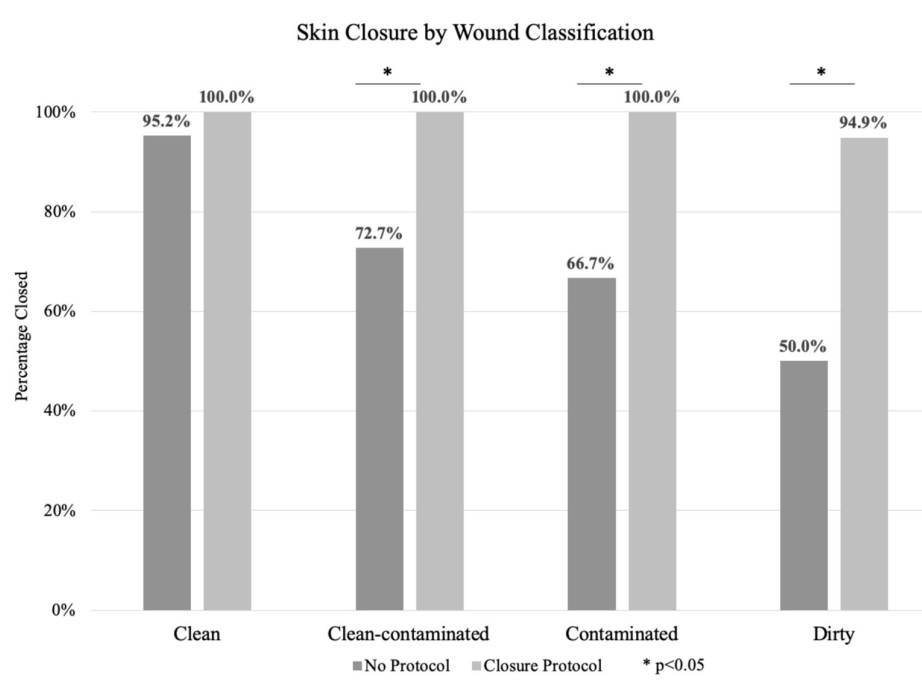

FIGURE 2: Skin Closure Rate by Wound Classification with and without Closure Protocol

A step-wise multivariate logistic regression evaluated the association of $\mathrm{CP}$ on skin closure with the inclusion of wound classification, BMI, ASA class, smoking, and initially open abdomen as possible confounders. CP significantly increased the likelihood of wound closure at discharge $(\mathrm{OR}=179.2 ; \mathrm{p}<0.001)$ (Table 4). Wound classification of contaminated or dirty and having an initially open abdomen were both negatively associated with skin closure. 


\section{Cureus}

\begin{tabular}{|c|c|c|c|}
\hline Variable & OR & 95\% Confidence Interval & p-value \\
\hline Closure protocol & 179.23 & 10.02-3206 & $<0.001$ \\
\hline Wound class $\geq$ III & 0.060 & $0.006-0.64$ & 0.02 \\
\hline Initially open & 0.028 & $0.027-0.28$ & 0.002 \\
\hline
\end{tabular}

TABLE 4: Logistic Regression Skin Closure

OR, odds ratio

\section{Discussion}

These results suggest that the use of a multi-modal incisional closure protocol in high-risk exploratory laparotomy patients utilizing iNPWT allows for the closure of nearly all wounds. Despite closing wounds that are high-risk for SSI, there was no difference in overall or superficial SSI rates in the CP group compared with the NP group. Furthermore, the rate of wound dehiscence was equivalent.

Retrospective studies have shown the benefit of iNPWT in reducing rates of SSI after laparotomy for gynecologic and general surgery patients [21,22]. However, the results of randomized controlled trials have been mixed. A randomized trial of iNPWT in obese patients undergoing cesarean reported a reduction in SSI rates [23]. Another randomized trial of elective and urgent general surgery patients showed lower rates of SSI with iNPWT, but patients with dirty wounds, BMI > 40, and ASA > III were excluded [24]. Randomized controlled trials of iNPWT in laparotomy for colorectal surgery or for intra-abdominal malignancy both showed no difference in the SSI rate $[25,26]$. Our study, similar to these randomized controlled trials that included high-risk patients undergoing laparotomy, showed no difference in the SSI rate with the use of iNPWT.

Although the alternative to iNPWT presented in most studies is a dry dressing over a closed incision, in emergent general surgery cases often the alternative is leaving the wound open to heal by secondary intention or performing delayed primary closure. A small randomized trial of iNPWT versus open NPWT after laparotomy in contaminated and dirty wounds showed no significant difference in SSI and more rapid healing in the iNPWT group [27]. A prospective study of iNPWT in high-risk emergent general surgery patients showed a superficial SSI rate of $7.4 \%$ [28], similar to the $8.4 \%$ rate seen in our study. In addition to SSI rates, it is important to consider wound closure as an outcome due to the increased cost of persistently open wounds. Acker et al. showed decreased SSI in trauma laparotomies that were allowed to heal by secondary intention, as well as higher costs [7]. In addition to direct costs, persistently open wounds may lead to indirect costs to patients and their families due to loss of independence, decreased mobility, and depression [29].

This study has multiple limitations. The data are retrospective and limited by what is documented in the chart. The external validity of the results is limited as the CP patients were operated on by a single surgeon at a single institution as part of a pilot study. Moreover, the use of a historical control group introduces the potential for confounding as other unknown factors in management may have changed, which could have affected the outcome of wound closure. However, a historical rather than concurrent control group was chosen because during the closure protocol time period, other surgeons in the department were adopting some aspects of the protocol but not others, which would have limited the ability to draw conclusions about the effects of the multi-modal protocol. As this study involves a multi-modal protocol, it is impossible to determine which aspects of the protocol lead to increased probability of closure. In the future, a multipronged randomized controlled trial will be required to determine which aspects of the closure protocol are necessary to prevent increased rates of SSI in high-risk groups.

\section{Conclusions}

By addressing both tissue factors and bacterial burden through the use of a multi-modal high-risk incisional closure protocol, all wounds can be considered for closure without increasing the risk of SSI or dehiscence. Given the limitations of this single-surgeon experience, further prospective study is needed.

\section{Additional Information}

\section{Disclosures}

Human subjects: Consent was obtained by all participants in this study. Washington University Institutional Review Board issued approval 201606040. Animal subjects: All authors have confirmed that this study did not involve animal subjects or tissue. Conflicts of interest: In compliance with the ICMJE uniform disclosure form, all authors declare the following: Payment/services info: All authors have 
declared that no financial support was received from any organization for the submitted work. Financial relationships: LJ Punch declare(s) personal fees from Acelity. Dr. Punch serves as a consultant for Acelity. Other relationships: All authors have declared that there are no other relationships or activities that could appear to have influenced the submitted work.

\section{Acknowledgements}

We would like to thank the entire acute care surgery team for their excellent patient care, which made this research possible.

\section{References}

1. Anderson DJ, Podgorny K, Berríos-Torres SI, et al.: Strategies to prevent surgical site infections in acute care hospitals: 2014 update. Infect Control Hosp Epidemiol. 2014, 35:605-627. 10.1086/676022

2. Alkaaki A, Al-Radi OO, Khoja A, et al.: Surgical site infection following abdominal surgery: a prospective cohort study. Can J Surg. 2019, 62:111-117. 10.1503/cjs.004818

3. Centers for Medicare and Medicaid Services (CMS), HHS: Medicare Program; hospital inpatient prospective payment systems for acute care hospitals and the long-term care hospital prospective payment system changes and FY2011 rates; provider agreements and supplier approvals; and hospital conditions of participation for rehabilitation and respiratory care services; Medicaid program: accreditation for providers of inpatient psychiatric services. Final rules and interim final rule with comment period. Federal Register. 2010, 75:50041-681.

4. Umscheid CA, Mitchell MD, Doshi JA, Agarwal R, Williams K, Brennan PJ: Estimating the proportion of healthcare-associated infections that are reasonably preventable and the related mortality and costs. Infect Control Hosp Epidemiol. 2011, 32:101-114. 10.1086/657912

5. Chetter IC, Oswald A V, Fletcher M, Dumville JC, Cullum NA: A survey of patients with surgical wounds healing by secondary intention; an assessment of prevalence, aetiology, duration and management. J Tissue Viability. 2017, 26:103-107. 10.1016/j.jtv.2016.12.004

6. He JC, Zosa BM, Schechtman D, et al.: Leaving the skin incision open may not be as beneficial as we have been taught. Surg Infect. 2017, 18:431-439. 10.1089/sur.2017.018

7. Acker A, Leonard J, Seamon MJ, et al.: Leaving contaminated trauma laparotomy wounds open reduces wound infections but does not add value. J Surg Res. 2018, 232:450-455. 10.1016/J.JSS.2018.05.083

8. Hepburn HH: Delayed primary suture of wounds. Br Med J. 1919, 1:181-183. 10.1136/bmj.1.3033.181

9. Duttaroy DD, Jitendra J, Duttaroy B, Bansal U, Dhameja P, Patel G, Modi N: Management strategy for dirty abdominal incisions: primary or delayed primary closure? A randomized trial. Surg Infect. 2009, 10:129-136. 10.1089/sur.2007.030

10. Siribumrungwong B, Chantip A, Noorit P, et al.: Comparison of superficial surgical site infection between delayed primary versus primary wound closure in complicated appendicitis: a randomized controlled trial. Ann Surg. 2018, 267:631-637. 10.1097/SLA.0000000000002464

11. Khan KI, Mahmood S, Akmal M, Waqas A: Comparison of rate of surgical wound infection, length of hospital stay and patient convenience in complicated appendicitis between primary closure and delayed primary closure. J Pak Med Assoc. 2012, 62:596-598.

12. Iqbal U, Green JB, Patel S, et al.: Preoperative patient preparation in enhanced recovery pathways. J Anaesthesiol Clin Pharmacol. 2019, 35:14-23.

13. Kelley KE, Fajardo AD, Strange NM, et al.: Impact of a novel preoperative patient-centered surgical wellness program. Ann Surg. 2018, 268:650-656. 10.1097/SLA.0000000000002932

14. Soper DE, Bump RC, Glenn Hurt W: Wound infection after abdominal hysterectomy: effect of the depth of subcutaneous tissue. Am J Obstet Gynecol. 1995, 173:465-471. 10.1016/0002-9378(95)90267-8

15. Gurunathan U, Ramsay S, Mitrić G, Way M, Wockner L, Myles P: Association between obesity and wound infection following colorectal surgery: systematic review and meta-analysis. J Gastrointest Surg. 2017, 21:1700-1712. 10.1007/s11605-017-3494-y

16. Winfield RD, Reese S, Bochicchio K, Mazuski JE, Bochicchio G V: Obesity and the risk for surgical site infection in abdominal surgery. Am Surg. 2016, 82:331-336.

17. Lambert KV, Hayes P, McCarthy M: Vacuum assisted closure: a review of development and current applications. Eur J Vasc Endovasc Surg. 2005, 29:219-226. 10.1016/j.ejvs.2004.12.017

18. McDonnell G, Russell AD: Antiseptics and disinfectants: activity, action, and resistance. Clin Microbiol Rev. 1999, 12:147-179.

19. Goztok M, Terzi MC, Egeli T, Arslan NC, Canda AE: Does wound irrigation with clorhexidine gluconate reduce the surgical site infection rate in closure of temporary loop ileostomy? A prospective clinical study. Surg Infect. 2018, 19:634-639. 10.1089/sur.2018.061

20. Surgical Site Infection (SSI) Event. (2019). Accessed: June 15, 2019: https://www.cdc.gov/nhsn/PDFs/PSCManual/9pscSSIcurrent.pdf.

21. Chambers LM, Morton M, Lampert E, et al.: Use of prophylactic closed incision negative pressure therapy is associated with reduced surgical site infections in gynecologic oncology patients undergoing laparotomy [Online ahead of print]. Am J Obstet Gynecol. 2020, 10.1016/j.ajog.2020.05.011

22. Zaidi A, El-Masry S: Closed-incision negative-pressure therapy in high-risk general surgery patients following laparotomy: a retrospective study. Colorectal Dis. 2017, 19:283-287. 10.1111/codi.13458

23. Hyldig N, Vinter CA, Kruse M, et al.: Prophylactic incisional negative pressure wound therapy reduces the risk of surgical site infection after caesarean section in obese women: a pragmatic randomised clinical trial. BJOG. 2019, 126:628-635. 10.1111/1471-0528.15413

24. O'Leary DP, Peirce C, Anglim B, et al.: Prophylactic negative pressure dressing use in closed laparotomy wounds following abdominal operations: a randomized, controlled, open-label trial: the P.I.C.O. trial. Ann Surg. 2017, 265:1082-1086. 10.1097/SLA.0000000000002098 


\section{Cureus}

25. Murphy PB, Knowles S, Chadi SA, et al.: Negative pressure wound therapy use to decrease surgical nosocomial events in colorectal resections (NEPTUNE): a randomized controlled trial. Ann Surg. 2019, 270:38-42. 10.1097/SLA.0000000000003111

26. Shen P, Blackham AU, Lewis S, et al.: Phase II randomized trial of negative-pressure wound therapy to decrease surgical site infection in patients undergoing laparotomy for gastrointestinal, pancreatic, and peritoneal surface malignancies. J Am Coll Surg. 2017, 224:726-737. 10.1016/j.jamcollsurg.2016.12.028

27. Frazee R, Manning A, Abernathy S, et al.: Open vs closed negative pressure wound therapy for contaminated and dirty surgical wounds: a prospective randomized comparison. J Am Coll Surg. 2018, 226:507-512. 10.1016/j.jamcollsurg.2017.12.008

28. Hall C, Regner J, Abernathy S, et al.: Surgical site infection after primary closure of high-risk surgical wounds in emergency general surgery laparotomy and closed negative-pressure wound therapy. J Am Coll Surg. 2019, 228:393-397. 10.1016/j.jamcollsurg.2018.12.006

29. McCaughan D, Sheard L, Cullum N, Dumville J, Chetter I: Patients' perceptions and experiences of living with a surgical wound healing by secondary intention: a qualitative study. Int J Nurs Stud. 2018, 77:29-38. 10.1016/j.ijnurstu.2017.09.015 